\title{
Long-term outcomes of endoscopic submucosal dissection versus surgery in early gastric cancer meeting expanded indication including undifferentiated-type tumors: a criteria-based analysis
}

\author{
Sunpyo Lee ${ }^{1} \cdot$ Kee Don Choi ${ }^{1} \cdot$ Minkyu Han $^{2} \cdot$ Hee Kyong Na ${ }^{1} \cdot J_{i}$ Yong Ahn ${ }^{1} \cdot$ \\ Kee Wook Jung ${ }^{1} \cdot$ Jeong Hoon Lee ${ }^{1} \cdot$ Do Hoon Kim $^{1} \cdot$ Ho June Song ${ }^{1} \cdot$ \\ Gin Hyug Lee ${ }^{1} \cdot J^{\prime}$ eong-Hwan Yook ${ }^{3} \cdot$ Byung Sik Kim $^{3} \cdot$ Hwoon-Yong Jung ${ }^{1}$
}

Received: 11 May 2017 / Accepted: 6 October 2017 / Published online: 19 October 2017

(C) The International Gastric Cancer Association and The Japanese Gastric Cancer Association 2017

\begin{abstract}
Background Endoscopic submucosal dissection (ESD) for early gastric cancer (EGC) meeting the expanded indication is considered investigational. We aimed to compare longterm outcomes of ESD and surgery for EGC in the expanded indication based on each criterion.

Methods This study included 1823 consecutive EGC patients meeting expanded indication conditions and treated at a tertiary referral center: 916 and 907 patients underwent surgery or ESD, respectively. The expanded indication included four discrete criteria: (I) intramucosal differentiated tumor, without ulcers, size $>2 \mathrm{~cm}$; (II) intramucosal differentiated tumor, with ulcers, size $\leq 3 \mathrm{~cm}$; (III) intramucosal undifferentiated tumor, without ulcers, size $\leq 2 \mathrm{~cm}$; and (IV) submucosal invasion $<500 \mu \mathrm{m}$ (sm1), differentiated tumor, size $\leq 3 \mathrm{~cm}$. We selected 522 patients in each group by propensity score matching and retrospectively evaluated each group. The primary outcome was overall survival (OS); the secondary outcomes were disease-specific survival (DSS), recurrence-free survival (RFS), and treatment-related complications.

Results In all patients and subgroups meeting each criterion, OS and DSS were not significantly different between groups (OS and DSS, all patients: $p=0.354$ and $p=0.930$;
\end{abstract}

Kee Don Choi

keedonchoi@gmail.com

1 Department of Gastroenterology, University of Ulsan College of Medicine, Asan Medical Center, Seoul, Korea

2 Department of Clinical Epidemiology and Biostatistics, University of Ulsan College of Medicine, Asan Medical Center, Seoul, Korea

3 Department of Surgery, University of Ulsan College of Medicine, Asan Medical Center, Seoul, Korea criteria I: $p=0.558$ and $p=0.688$; criterion II: $p=1.000$ and $p=1.000$; criterion III: $p=0.750$ and $p=0.799$; and criterion IV: $p=0.599$ and $p=0.871$ ). RFS, in all patients and criterion I, was significantly shorter in the ESD group than in the surgery group $(p<0.001$ and $p<0.003$, respectively). The surgery group showed higher rates of late and severe treatment-related complications than the ESD group. Conclusions ESD may be an alternative treatment option to surgery for EGCs meeting expanded indications, including undifferentiated-type tumors.

Keywords Early gastric cancer - Expanded indication . Endoscopic submucosal dissection · Gastrectomy · Longterm outcome

\section{Introduction}

Conventional gastrectomy with lymph node dissection is accepted as a standard treatment for early gastric cancer (EGC). However, in the past two decades, many studies have shown favorable long-term outcomes of endoscopic resection (ER) [1, 2]. As a result, ER, including endoscopic mucosal resection and endoscopic submucosal dissection (ESD), became a standard treatment for tumors meeting the specific criteria characteristic of very low lymph node metastasis risk: intramucosal differentiated-type adenocarcinoma and $\leq 2 \mathrm{~cm}$ in size with no ulcers [3]. With the accumulation of experience and technical advances, the indication for ER has widened, and expanded indication criteria were proposed based on the minimal risk of lymph node metastasis. The expanded indication consists of four discrete criteria: criterion I, intramucosal tumor, differentiated type, without ulcerative findings, and $>2 \mathrm{~cm}$ in size; criterion II, intramucosal tumor, differentiated type, with 
ulcerative findings, and $\leq 3 \mathrm{~cm}$ in size; criterion III, intramucosal tumor, undifferentiated type, without ulcerative findings, and $\leq 2 \mathrm{~cm}$ in size; and criterion IV, submucosal invasion $<500 \mu \mathrm{m}$ ( $\mathrm{sm} 1)$, differentiated type, and $\leq 3 \mathrm{~cm}$ in size. Previous studies investigated the long-term outcomes of ESD in EGCs meeting the expanded indication criteria, and the results were comparable with those in patients meeting the absolute indication criteria [1, 4]. Recently, to validate the expanded indication criteria of ESD, several studies compared the long-term outcomes of ESD and gastrectomy, reporting favorable results of ESD [4-6]. However, there have been several case reports of lymph node or distant metastases after curative ESD in EGC patients meeting the expanded indication, and ESD is still under investigation for this indication [7-10]. In the expanded indication criteria, evidence is still insufficient for undifferentiated-type tumors and superficially invasive submucosal tumors. As the expanded indication criteria are diverse in character, the prognosis could be different for each criterion after ESD. Previous studies commonly excluded undifferentiated-type tumors and included only a very small number of submucosal cancers in their analyses. In this study, we evaluated and compared the long-term outcomes of ESD and surgery, based on the entire indication and individual criteria, for EGCs meeting the expanded indication, including undifferentiated-type tumors.

\section{Methods}

\section{Study population and data collection}

All patients diagnosed with EGC who underwent ESD between January 2005 and June 2013 or surgery between January 2003 and December 2011 at Asan Medical Center and met the curative resection criteria for tumors of expanded indication in the post-procedural pathology report were retrospectively enrolled in this study. The inclusion criteria were (1) age $\geq 20$ years, (2) newly diagnosed EGC meeting the curative resection criteria for tumors of expanded indication (criterion I: intramucosal tumor, differentiated type, without ulcerative findings, and $>2 \mathrm{~cm}$ in size; criterion II: intramucosal tumor, differentiated type, with ulcerative findings, and $\leq 3 \mathrm{~cm}$ in size; criterion III: intramucosal tumor, undifferentiated type, without ulcerative findings, and $\leq 2 \mathrm{~cm}$ in size; and criterion IV: submucosal invasion $<500 \mu \mathrm{m}(\mathrm{sm} 1)$, differentiated type, and $\leq 3 \mathrm{~cm}$ in size), and (3) follow-up duration $\geq 1$ year. The exclusion criteria were (1) multiple EGCs or synchronous EGCs, (2) previous or concurrent history of malignancies of other organs, except for fully recovered thyroid cancer, cervical carcinoma in situ, and nonmetastatic prostate cancer, (3) previous gastric cancer treatment, including neoadjuvant chemotherapy and ER, (4) adjuvant chemotherapy after surgery, and (5) consecutive gastrectomy with lymph node dissection within 1 year after ESD. This study was approved by the Institutional Review Board at Asan Medical Center, Korea (2016-0896).

Patient clinical data, including age, sex, comorbidities, age-adjusted Charlson Comorbidity index [11], tumor characteristics such as size, morphology, location, depth of invasion, histology, presence of lymphovascular invasion, complications, recurrence, and mortality, were collected by reviewing medical records. Tumor stage was determined according to the American Joint Committee on Cancer classification system, 7 th edition. Complications were classified according to the Clavien-Dindo classification [12], which consisted of five severity grades as follows: grade 1, any deviation from the normal postoperative course, without the need for pharmacological treatment or surgical, endoscopic, or radiological interventions; grade 2 , requiring pharmacological treatment with drugs other than those allowed for grade 1 complications, requiring blood transfusions, or total parenteral nutrition; grade 3a, requiring surgical, endoscopic, or radiological intervention not under general anesthesia; grade $3 \mathrm{~b}$, requiring interventions as described for Grade $3 \mathrm{a}$ but under general anesthesia; grade 4 , life-threatening complication requiring intermediate care/intensive care unit management; grade 5 , death.

\section{Treatment procedures and follow-up protocol}

ESD was performed under sedation with intravenous midazolam $(0.05 \mathrm{mg} / \mathrm{kg})$ and pethidine $(50 \mathrm{mg})$. The typical ESD procedures at our institution included marking, mucosal incision, and submucosal dissection with simultaneous hemostasis, and have been previously described [1]. The surgical procedure, radical gastrectomy with more than D1+ beta lymph node dissection, was performed, consistent with the recommendations of the Japanese Gastric Cancer Treatment Guidelines [3]. Total, distal, or proximal gastrectomy was performed by experienced surgeons based on location. In the ESD group, patients were checked for Helicobacter pylori infection by rapid urease tests, urea breath tests, or histopathological examination, and infected patients underwent eradication therapy after ESD. In the surgery group, however, tests for Helicobacter pylori infection and subsequent eradication treatment were not performed mandatorily. After ESD and surgery, follow-up esophagogastroduodenoscopy and abdominal computed tomography were performed every 6 months for the first 2 years and then annually for the next 3 years. 


\section{Histological examination}

The resected specimens were stretched, pinned to a polystyrene plate, and totally immersed in $10 \%$ neutral buffered formalin. After fixation, the specimens were grossly examined to identify lesions and the closest resection margins. Surgical specimens were sectioned into 2-mm-thick slices. ER specimens were sectioned into 2-mm-thick slices parallel to an imaginary line drawn from the edge of the tumor to the closest resection margin after applying black ink to all lateral and deep resection margins. Each sliced tissue specimen was embedded in paraffin, and 5- $\mu$ m sections were cut from each paraffin block and stained with hematoxylin and eosin. Lymph nodes were cut into two pieces and the cut surfaces were examined to determine the status of the nodes. Lymphovascular invasion was defined as the observable spread of tumor cells through the lymphatic vessels (e.g., carcinoma cells floating within the endothelia-lined space). Tumors were histologically classified according to the World Health Organization Classification of Tumors [13].

\section{Outcome assessment}

The primary outcome of this study was overall survival (OS). Secondary outcomes included disease-specific survival (DSS), recurrence-free survival (RFS), and treatmentrelated complications. Patient survival status was obtained from the claims database of the Korean National Health Insurance Corporation. Follow-up data on deaths and recurrences until June 2016 were collected from patient medical records. OS was measured from the date of ESD or surgery until death from any cause, and DSS was measured from the date of ESD or surgery until death resulting from gastric cancer. If the cause of death was not known because of loss to follow-up, the case was designated as death from gastric cancer. RFS was measured from the date of ESD or surgery until the first recurrence or death from any cause. Metachronous recurrence was defined as a new cancer at a previously uninvolved site in the remnant stomach occurring more than 1 year after treatment.

\section{Statistical analysis}

Continuous variables are presented as mean \pm standard deviation or median with interquartile range (IQR). Comparisons of categorical variables were performed using the chi-square or Fisher's exact tests, and comparisons of continuous variables were performed using the Student's $t$ test or Mann-Whitney $U$ test. To reduce selection bias, we performed a propensity score-matching analysis between ESD and surgery groups. The propensity scores were estimated from a logistic regression model by including variables, such as age, sex, comorbidities, expanded indication criteria, as well as size, depth, location, morphology, and histology of tumors, which could potentially affect the outcome of treatment. Using these propensity scores, patients in the ESD group were matched at a one-to-one ratio with those in the surgery group. The Kaplan-Meier analysis was used to estimate survival differences between the two treatment methods in all patients and in each expanded indication criteria group. Statistical significance was assessed using the logrank test. The hazard ratios (HR) for long-term outcomes of ESD over surgery in all patients and within each subgroup were estimated using the Cox proportional hazard regression model. All data were analyzed using SPSS for Windows statistical software package (version 21.0; SPSS, Chicago, IL, USA) and the R statistical package (V.3.1.2; free download from http://www.r-project.org). $p$ values $<0.05$ were considered statistically significant.

\section{Results}

\section{Demographic and baseline characteristics}

During the study period, 1336 patients who met the expanded indication criteria underwent ESD and 1493 patients underwent surgery for EGCs. A total of 907 patients in the ESD group and 916 patients in the surgery group were included in this study based on our predetermined inclusion and exclusion criteria (Fig. 1). Baseline characteristics of all patients and propensity score-matched patients are shown in Table 1. The median follow-up period was 58 months (IQR, 38.6-66); 50.2 months (IQR, 36.6-64.9) in the ESD group and 59.5 months (IQR, 49.4-67.7) in the surgery group. The 5-year adherence rates to follow-up were $32.9 \%$ for the ESD group and $46.1 \%$ for the surgery group. This difference was not statistically significant. The median age and the rate of underlying diseases were significantly higher in the ESD group than in the surgery group, whereas the rates of undifferentiated histology and flat/depressed morphology were significantly higher in the surgery group. The distribution of patients for each expanded indication criterion was significantly different between the two treatment groups $(p<0.001)$. The proportion of patients meeting criterion I was much higher in the ESD group than in the surgery group, whereas the proportion of patients meeting criterion III was much higher in the surgery group than in the ESD group. The rate of lymphovascular invasion was similar between the groups. In the ESD group, the involvement of resection margin was identified in $3.3 \%$ of patients $(n=30)$, and the curative resection rate was $95.7 \%(n=868)$. In the surgery group, subtotal and total gastrectomy were performed in $85 \%(n=779)$ and $15 \%(n=137)$ of patients, respectively. Open surgery was performed in $65.8 \%(n=603)$ and laparoscopic surgery in $34.2 \%(n=313)$ of patients. Lymph 


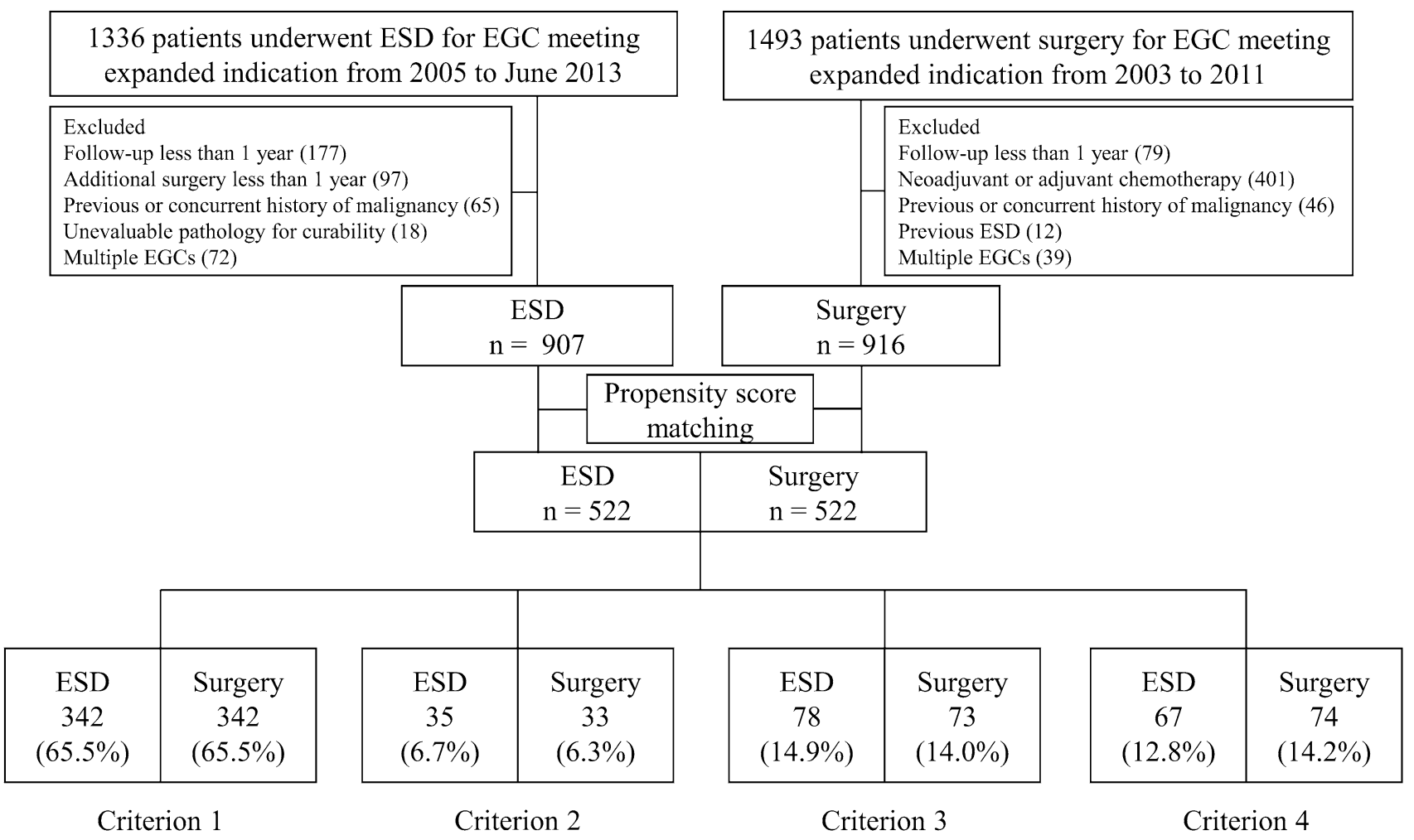

Fig. 1 Study flowchart

node metastasis was discovered in 13 patients (1.4\%). After propensity score matching, 522 patients in each treatment group were analyzed; there were no statistical differences in demographic and baseline characteristics between the two groups.

\section{Comparison of long-term outcomes between endoscopic resection and surgery}

\section{Overall survival}

Kaplan-Meier OS curves are presented in Fig. 2a. The 5-year OS rates for the ESD and surgery groups were $97.1 \%$ and $97.6 \%$, respectively, and the ESD group appeared to be associated with worse prognosis [hazard ratio (HR): 2.404, 95\% confidence interval (CI), $1.635-3.535, p<0.001]$. However, after propensity score matching, the 5-year OS rates were $98.1 \%$ in the ESD group and $96.4 \%$ in the surgery group, and no correlation between ESD and poor prognosis was observed (HR: $1.253,95 \%$ CI, 0.777-2.023, $p=0.355)$. For criteria I and III subgroups, OS was not significantly different between ESD and surgery patients (criterion 1, HR: 1.193, 95\% CI, 0.661-2.151, $p=0.558$; criterion III, HR: $1.341,95 \% \mathrm{CI}$, $0.219-8.221, p=0.751)$. For criterion II, no mortalities were reported in the surgery group, and HR was not evaluable. However, Kaplan-Meier analysis suggested no significant differences in OS between the two treatment groups $(p=0.750)$. For the criterion IV subgroup, no significant differences were detected in OS between groups (HR: $1.277,95 \%$ CI, 0.513-3.179, $p=0.600$ ).

\section{Disease-specific survival}

After propensity score matching, 5-year DSS rates were 99.6\% and $98.9 \%$ in the ESD and surgery groups, respectively. Kaplan-Meier analysis of DSS showed no significant differences between the groups $(p=0.931)$, and the HR for disease-specific mortality in the ESD group appeared lower than that of the surgery group (HR: 0.846, 95\% CI, 0.374-1.915, $p=0.930$ ). For the DSS of criteria I, III, and IV subgroups, the ESD group appeared to have a more favorable prognosis than the surgery group, but these differences were not statistically significant (criterion I, HR: $0.846,95 \%$ CI, 0.374-1.915, $p=0.689$; criterion III, HR: $0.733,95 \%$ CI, 0.066-8.084, $p=0.800$; criterion IV, HR: $0.901,95 \%$ CI, 0.258-3.15, $p=0.871)$. For criterion II subgroups, Kaplan-Meier analysis showed no significant differences between the two groups $(p=1.000)$. 
Table 1 Baseline clinicopathological characteristics of the study patients grouped by treatment method before and after propensity score matching

\begin{tabular}{|c|c|c|c|c|c|c|}
\hline & \multicolumn{3}{|l|}{ Overall } & \multicolumn{3}{|c|}{ Propensity score matching } \\
\hline & $\operatorname{ESD}(n=907)$ & Surgery $(n=916)$ & $p$ & $\operatorname{ESD}(n=522)$ & Surgery $(n=522)$ & $p$ \\
\hline Age & $64(56-70)$ & $57.5(49-66)$ & $<0.001$ & $61(53-68)$ & $61(54-68)$ & 0.566 \\
\hline Sex, $n(\%)$ & & & $<0.001$ & & & 0.839 \\
\hline Male & $662(73.0)$ & $566(61.8)$ & & $366(70.1)$ & $370(70.9)$ & \\
\hline Female & $245(27.0)$ & $350(38.2)$ & & $156(29.9)$ & $152(29.1)$ & \\
\hline CCI, IQR & $2(1-3)$ & $1(0-2)$ & $<0.001$ & $2(1-3)$ & $2(1-3)$ & 0.834 \\
\hline Comorbidities, $n(\%)$ & $458(50.5)$ & $327(35.7)$ & $<0.001$ & $220(42.2)$ & $232(44.4)$ & 0.492 \\
\hline Diabetes mellitus & $129(14.2)$ & $89(9.7)$ & 0.004 & $65(12.5)$ & $63(12.1)$ & 0.925 \\
\hline Hypertension & $350(38.6)$ & $234(25.5)$ & $<0.001$ & $163(31.2)$ & $172(33.0)$ & 0.596 \\
\hline Liver cirrhosis CPS A/CPS B & $8(0.9) / 6(0.7)$ & $6(0.7) / 0(0)$ & 0.041 & $1(0.2) / 1(0.2)$ & $5(1.0) / 0(0)$ & 0.159 \\
\hline Heart failure & $3(0.3)$ & 0 & 0.245 & 0 & 0 & 1.000 \\
\hline Coronary artery disease & $22(2.4)$ & $19(2.1)$ & 0.728 & $11(2.1)$ & $15(2.9)$ & 0.551 \\
\hline COPD & $10(1.1)$ & $3(0.3)$ & 0.091 & $2(0.4)$ & $3(0.6)$ & 1.000 \\
\hline Chronic kidney disease & $6(0.7)$ & $5(0.5)$ & 0.987 & $4(0.8)$ & $2(0.4)$ & 0.682 \\
\hline Cerebrovascular disease & $20(2.2)$ & $12(1.3)$ & 0.202 & $9(1.7)$ & $8(1.5)$ & 1.000 \\
\hline Size & $25(21-33)$ & $22(15-32.5)$ & $<0.001$ & $25(20-35)$ & $25(21-35)$ & 0.503 \\
\hline Morphology, $n(\%)$ & & & $<0.001$ & & & 1.000 \\
\hline Elevated & $251(27.7)$ & $164(17.9)$ & & $127(24.3)$ & $128(24.5)$ & \\
\hline Flat/depressed & $656(72.3)$ & $752(82.1)$ & & $395(75.7)$ & $394(75.5)$ & \\
\hline Histology, $n(\%)$ & & & $<0.001$ & & & 0.738 \\
\hline Well differentiated & $478(52.7)$ & $236(25.8)$ & & $207(40.0)$ & $203(38.9)$ & \\
\hline Moderately differentiated & $346(38.2)$ & $340(37.1)$ & & $237(45.4)$ & $247(47.3)$ & \\
\hline Poorly differentiated & $56(6.2)$ & $167(18.2)$ & & $52(10.0)$ & $43(8.2)$ & \\
\hline Signet-ring cell & $27(3.0)$ & $173(18.9)$ & & $26(5.0)$ & $29(5.6)$ & \\
\hline Depth, $n(\%)$ & & & 0.882 & & & 0.587 \\
\hline Mucosa & $807(89.0)$ & $818(89.3)$ & & $455(87.2)$ & $448(85.9)$ & \\
\hline Submucosa & $100(11.0)$ & $98(10.7)$ & & $67(12.8)$ & $74(14.2)$ & \\
\hline Location, $n(\%)$ & & & 0.008 & & & 0.549 \\
\hline Upper third & $67(7.4)$ & $65(7.1)$ & & $36(6.9)$ & $43(8.2)$ & \\
\hline Mid-third & $191(21.0)$ & $250(27.3)$ & & $114(21.8)$ & $122(23.4)$ & \\
\hline Lower third & 649 (71.6) & $601(65.6)$ & & $372(71.3)$ & $357(68.4)$ & \\
\hline Lymphovascular invasion, $n(\%)$ & $9(1)$ & $11(1.2)$ & 0.669 & $7(1.3)$ & $8(1.5)$ & 0.795 \\
\hline Lymph node involvement, $n(\%)$ & - & $13(1.4)$ & & - & $7(1.3)$ & \\
\hline Resection margin $(\mathrm{V} / \mathrm{H})$ & $27(3) / 3(0.3)$ & - & & $20(3.8) / 3(0.6)$ & - & \\
\hline Curative resection & $868(95.7)$ & - & & $492(94.3)$ & - & \\
\hline Criteria of expanded indication & & & $<0.001$ & & & 0.903 \\
\hline Criterion $1, n(\%)$ & $660(72.8)$ & $431(47.1)$ & & $342(65.5)$ & $342(65.5)$ & \\
\hline Criterion $2, n(\%)$ & $64(7.1)$ & $46(5.0)$ & & $35(6.7)$ & $33(6.3)$ & \\
\hline Criterion $3, n(\%)$ & $83(9.2)$ & $341(37.2)$ & & 78 (14.9) & $73(14.0)$ & \\
\hline Criterion $4, n(\%)$ & $100(11.0)$ & $98(10.7)$ & & $67(12.8)$ & $74(14.2)$ & \\
\hline Follow-up duration, median (IQR), month & $50.2(36.6-64.9)$ & $59.5(49.4-67.7)$ & $<0.001$ & $52.7(37.7-67.9)$ & $59.2(47.9-63.4)$ & 0.008 \\
\hline
\end{tabular}

$n$ number, $C O P D$ chronic obstructive pulmonary disease, $I Q R$ interquartile range, $C C I$ age-adjusted Charlson comorbidity index, $C P S$ ChildPugh score, $V$ vertical, $H$ horizontal

\section{Recurrence-free survival}

After propensity score matching, 5-year RFS rates were $92.7 \%$ and $96.7 \%$ in the ESD and surgery groups, respectively. RFS was significantly lower in the ESD group than in the surgery group (HR: $1.916,95 \%$ CI, $1.319-2.782, p<0.001)$. For criterion I subgroups, RFS was lower in the ESD group than in the surgery group 

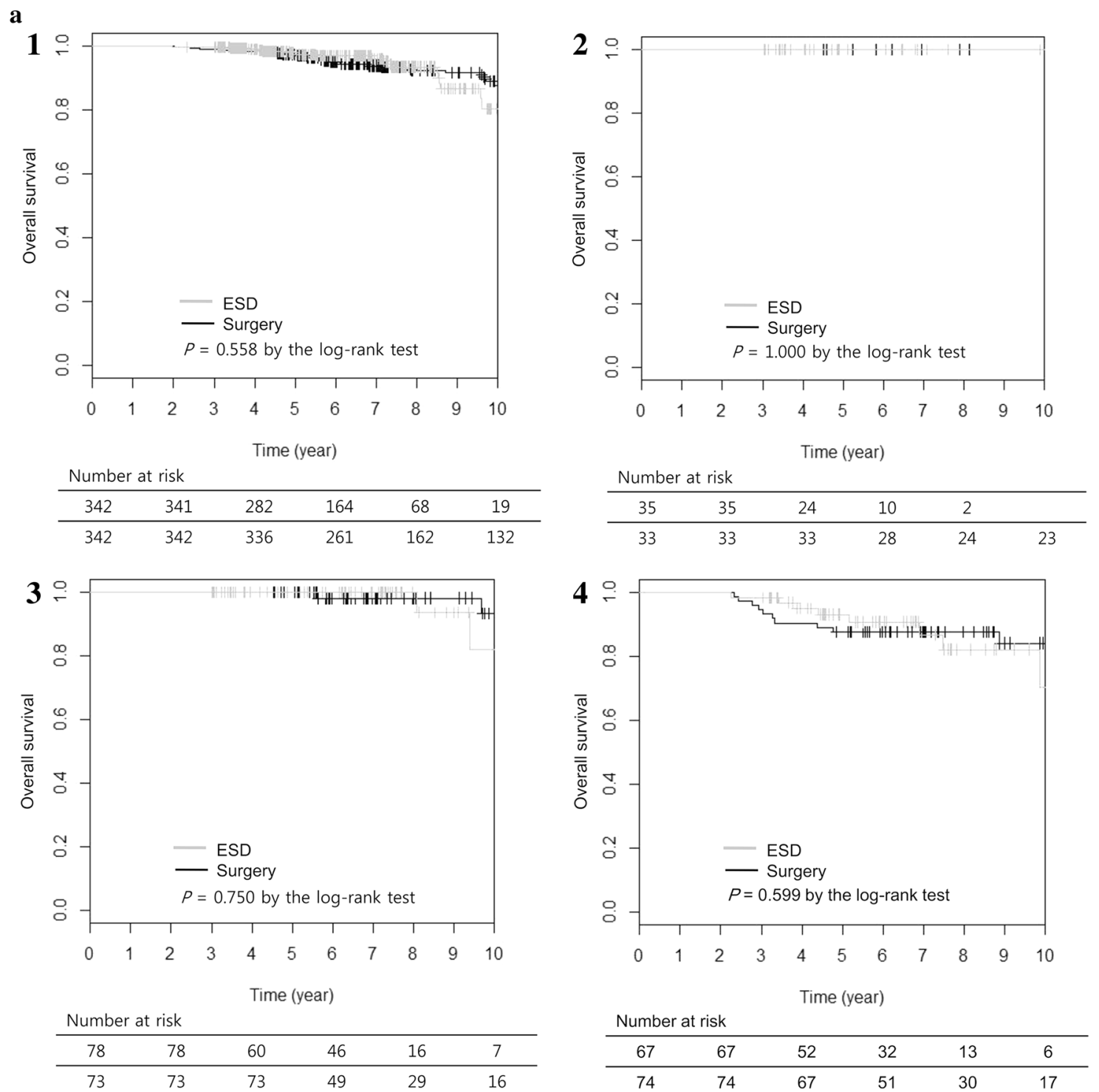

Fig. 2 Kaplan-Meier survival curves for endoscopic resection and surgery groups before and after matching. a Overall survival: $a-1$ Criterion I, $a$-2 Criterion II, $a$-3 Criterion III, $a-4$ Criterion IV. b Recur-

rence-free survival: $b$ - 1 Criterion I, $b$-2 Criterion II, $b$-3 Criterion III, $b-4$ Criterion IV. Gray line ESD group, black line surgery group

$(p=0.003)$. Although the surgery group appeared to have an RFS benefit compared with the ESD group for criteria II, III, and IV subgroups as well, the differences between groups were not significant (criterion II, HR: 4.662, 95\% CI, 0.478-45.47, $p=0.185$; criterion III, HR: 2.848, 95\% CI, 0.766-10.590, $p=0.118$; criterion IV, HR: $1.146,95 \%$ CI, 0.414-3.172, $p=0.792$ ) (Fig. 2b). Table 2 shows recurrence patterns after treatment in EGC patients meeting the expanded indication criteria. Overall, the ESD group had more recurrences than did the surgery group ( $8.7 \%$ and $1.7 \%$, respectively; $p<0.001)$. However, metachronous recurrences comprised $84.4 \%$ of recurrences in the ESD group, and no distant recurrences

were reported. ESD was the salvage treatment most commonly chosen for local and metachronous recurrences and had an $88.9 \%$ cure rate; $15.6 \%$ of recurrences were successfully treated with gastrectomy. In the surgery group, metachronous recurrence was not the dominant recurrence type. Extragastric recurrences accounted for $33.3 \%$ of relapses in this group and caused a fatal outcome. Excluding metachronous recurrences, we compared RFS of the two treatment groups via Kaplan-Meier analysis. There were no significant differences in RFS between the groups in all patients or in any of the subgroups by each criterion (all patients, $p=0.642$; criterion I, $p=0.746$; criterion II, $p=0.332$; criterion III, $p=0.068$; criterion $\mathrm{IV}, p=0.947)$. 

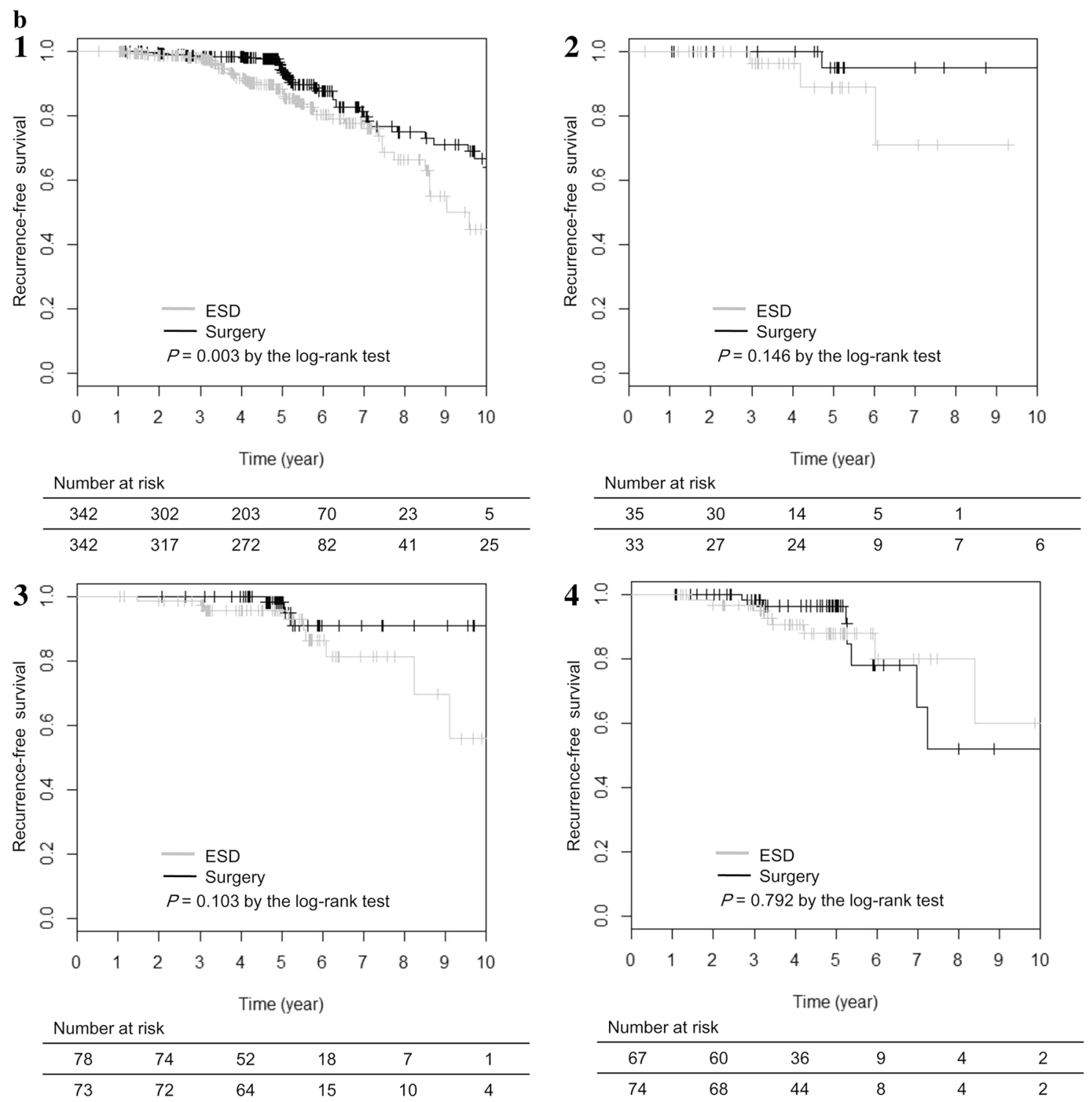

Fig. 2 (continued)

\section{Treatment-related complications}

Complications encountered in both groups are presented in Table 3. Overall, 37 patients (4.1\%) in the ESD group and 45 patients $(4.9 \%)$ in the surgery group encountered complications $(p=0.388)$. Criterion IV subgroups had the highest complication rates for both treatments: $6.0 \%$ in the ESD group and $11.2 \%$ in the surgery group. The most common complications were bleeding (64.9\%) in the ESD group and mechanical ileus (48.9\%) in the surgery group. Post-ESD bleeding and post-ESD perforation occurred most frequently in the criterion IV subgroups $(3.0 \%, p=0.699$ and 3\%, $p=0.005$, respectively). Mechanical ileus also developed most frequently in criterion IV subgroups $(7.1 \%, p=0.069)$, but was also significantly related to total gastrectomy $(p<0.001)$. In the ESD group, all complications occurred within 1 month after ESD, 31 (83.8\%) of which were successfully treated with an endoscopic procedure (Clavien-Dindo classification grade $3 \mathrm{~A}$ ). In the surgery group, 16 complications (35.6\%) arose 1 month after surgery, 14 of which were mechanical ileus. Overall, the surgery group alone had the complications requiring an operation or more (Clavien-Dindo classification grade 3B or more), and there were no complication-related deaths.

\section{Discussion}

In the present study, using a propensity score-matching analysis of EGC that met the expanded indication criteria, 
Table 2 Recurrence pattern and treatment after ESD and surgery for early gastric cancer meeting expanded indication criteria

\begin{tabular}{|c|c|c|c|c|c|c|}
\hline & \multicolumn{3}{|l|}{ Overall } & \multicolumn{3}{|c|}{ Propensity score matching } \\
\hline & $\operatorname{ESD}(n=907)$ & Surgery $(n=916)$ & $p$ & $\operatorname{ESD}(n=522)$ & Surgery $(n=522)$ & $p$ \\
\hline Recurrence & $75(8.3)$ & $11(1.2)$ & $<0.001$ & $45(8.7)$ & $9(1.7)$ & $<0.001$ \\
\hline Local & $10(1.1)$ & $4(0.4)$ & & $5(1.0)$ & $3(0.6)$ & \\
\hline Metachronous & $63(6.9)$ & $4(0.4)$ & & $38(7.3)$ & $3(0.6)$ & \\
\hline Regional lymph node & $2(0.2)$ & $2(0.2)$ & & $2(0.4)$ & $2(0.4)$ & \\
\hline Distant & $0(0)$ & $1(0.1)$ & & $0(0)$ & $1(0.2)$ & \\
\hline Interval to recurrence, months & $31(18.5-53.5)$ & $37.8(24.7-61)$ & 0.974 & $30.7(17.4-57.8)$ & $38.6(21.9-63)$ & 0.818 \\
\hline \multicolumn{7}{|l|}{ Treatment after recurrence } \\
\hline ESD & $55(6.1)$ & $2(0.2)$ & & $32(6.1)$ & $2(0.4)$ & \\
\hline Surgery & $7(0.8)$ & $5(0.5)$ & & $3(0.6)$ & $4(0.8)$ & \\
\hline Noncurative ESD and surgery & $6(0.7)$ & - & & $4(0.8)$ & - & \\
\hline Chemotherapy & - & $3(0.3)$ & & - & $2(0.4)$ & \\
\hline No treatment & $3(0.3)$ & $1(0.1)$ & & $3(0.6)$ & $1(0.2)$ & \\
\hline No information & $4(0.4)$ & - & & $3(0.6)$ & - & \\
\hline
\end{tabular}

$n$ number, ESD endoscopic submucosal dissection

Table 3 Treatment-related complications grouped by treatment method for early gastric cancer before and after propensity score matching

\begin{tabular}{|c|c|c|c|c|c|c|}
\hline & \multicolumn{3}{|l|}{ Overall } & \multicolumn{3}{|c|}{ Propensity score matching } \\
\hline & $\operatorname{ESD}(n=907)$ & Surgery $(n=916)$ & $p$ & $\operatorname{ESD}(n=522)$ & Surgery $(n=522)$ & $p$ \\
\hline Complications & $37(4.1)$ & $45(4.9)$ & 0.388 & $21(4.0)$ & $31(5.9)$ & 0.155 \\
\hline Post-ESD bleeding & $24(2.6)$ & & & $13(2.5)$ & & \\
\hline Post-ESD perforation & $8(0.9)$ & & & $5(1.0)$ & & \\
\hline Post-ESD stricture & $3(0.3)$ & & & $2(0.4)$ & & \\
\hline Pneumonia & $2(0.2)$ & $2(0.2)$ & & $1(0.2)$ & $2(0.3)$ & \\
\hline Ileus & & $22(2.4)$ & & & $13(2.5)$ & \\
\hline Anastomotic bleeding & & $9(1.0)$ & & & $7(1.3)$ & \\
\hline Intraabdominal bleeding & & $1(0.1)$ & & & $1(0.2)$ & \\
\hline Anastomotic leakage & & $4(0.4)$ & & & $3(0.6)$ & \\
\hline Anastomotic stricture & & $1(0.1)$ & & & $0(0)$ & \\
\hline Postoperative abscess & & $2(0.2)$ & & & $2(0.4)$ & \\
\hline Incisional hernia & & $1(0.1)$ & & & $1(0.2)$ & \\
\hline Wound problem & & $3(0.3)$ & & & $2(0.4)$ & \\
\hline Criteria of expanded indication & & & 0.010 & & & 0.599 \\
\hline Criterion I & $27(3.0)$ & $18(2.0)$ & & $13(2.5)$ & $17(3.3)$ & \\
\hline Criterion II & $1(0.1)$ & $2(0.2)$ & & $1(0.2)$ & $2(0.4)$ & \\
\hline Criterion III & $3(0.3)$ & $14(1.5)$ & & $3(0.6)$ & $4(0.8)$ & \\
\hline Criterion IV & $6(0.7)$ & $11(1.2)$ & & $4(0.8)$ & $8(1.6)$ & \\
\hline Time to complication & & & $<0.001$ & & & 0.006 \\
\hline Within 1 month & $37(4.1)$ & $29(3.2)$ & & $21(4.0)$ & $21(4.0)$ & \\
\hline After 1 month & $0(0)$ & $16(1.7)$ & & & $10(1.9)$ & \\
\hline Clavien-Dindo classification & & & $<0.001$ & & & $<0.001$ \\
\hline Grade 1 & $1(0.1)$ & $0(0)$ & & $1(0.2)$ & $0(0)$ & \\
\hline Grade 2 & $5(0.6)$ & $25(2.7)$ & & $1(0.2)$ & $17(3.3)$ & \\
\hline Grade $3 \mathrm{~A}$ & $31(3.4)$ & $10(1.0)$ & & $19(3.6)$ & $6(1.1)$ & \\
\hline Grade $3 \mathrm{~B}$ or more & 0 & $10(1.2)$ & & 0 & $8(1.6)$ & \\
\hline Hospital days, IQR & $4(4-5)$ & $10(9-11)$ & $<0.001$ & $4(4-5)$ & $10(9-11.3)$ & $<0.001$ \\
\hline
\end{tabular}

$n$ number, $E S D$ endoscopic submucosal dissection, $I Q R$ interquartile range 
OS and DSS were not significantly different between the ESD and surgery groups. Similarly, when OS and DSS were analyzed based on each criterion, no differences were observed between the treatment groups. The RFS was significantly lower in the ESD group than in the surgery group for patients meeting criterion I (intramucosal differentiated tumor, without ulcers, size $>2 \mathrm{~cm}$ ). Excluding metachronous recurrences, the RFS was not significantly different between the two groups. The ESD group had significantly more metachronous recurrences than the surgery group, but no distant recurrences were detected. All the treatment-related complications in the ESD group were encountered within 1 month after the procedure and could be managed with either endoscopic or conservative treatment. However, in the surgery group, one third of complications arose 1 month after surgery, and one fourth of complications required reoperation.

Several researchers have reported the feasibility of ESD in EGCs meeting the expanded indication by several different analyses. Park et al. compared long-term outcomes of ESD for EGCs that met the expanded indication with EGCs which met the absolute indication criteria [14]. Hasuike et al. evaluated the efficacy and safety of ESD for intramucosal gastric cancer meeting the expanded indication in a multi-center single-arm confirmatory trial [15], and Fukunaga et al. compared long-term prognosis of ESD versus surgery in EGCs that met the expanded indication; however, they included only differentiated-type EGCs [5]. Kim et al. analyzed the outcomes of each treatment method based on the individual criteria in the expanded indication, but they excluded undifferentiated-type EGCs as well [16]. Other studies included only undifferentiated-type EGCs among the four criteria of the expanded indication for evaluation of the outcomes of ESD [17]. Those recent studies showed comparable outcomes of ESD in EGCs that met the expanded indication; however, none of them included all EGCs meeting each criterion of the expanded indication or examined the outcomes of EGCs by individual criterion. However, in our study, we included all EGCs that met each criterion of the expanded indication and analyzed the long-term treatment outcomes of the EGCs in subgroups of patients who met each criterion. Our study found that OS and DSS were not significantly different between the groups based on individual criteria.

In the current study, similar to that which was observed in previous studies $[1,4,16]$, the most common recurrence type in the ESD group was metachronous recurrence (7.3\%). Repeated ESD was an effective treatment method for local and metachronous recurrences, and surgery was a successful treatment approach as well, especially in cases of regional lymph node recurrence. Metachronous recurrence was not a predominant recurrence type in the surgery group; however, all cases of metachronous recurrence could also be successfully treated by ESD or reoperation. In the surgery group, there were three cases of local recurrence at the anastomotic site, which were treated with rescue surgery, and three cases of extragastric recurrence, resulting in a fatal outcome from disease progression. Excluding metachronous recurrences, RFS was not significantly different between the two treatment groups for the entire expanded indication population and for each criterion. Several studies have reported that metachronous recurrence could be effectively detected by endoscopic surveillance in the early stage and cured by repeated ESD and surgery [10, 18, 19]. Thus, with scheduled and thorough endoscopic surveillance and rescue ESD, the effects of metachronous recurrence on survival can be favorably managed.

The present study had several limitations. First, this study has a retrospective design; thus, randomization to two treatment groups could not be achieved. To overcome this limitation, we employed propensity score-matching analysis and tried to minimize the differences in clinicopathological characteristics between the two groups. Second, the study period for the ESD group was 2 years later than that of the surgery group. To identify reliable long-term outcomes, we planned at least a 5-year follow-up period and collected data from the patients who underwent gastrectomy between 2003 and 2011. Because ESD was not introduced to our center until 2005, we were unable to assess outcomes of ESD within the same timeframe. To minimize the effects of this limitation, we included patients who underwent ESD between 2005 and 2014 and maintained the same study duration of 9 years. In addition, this is the largest study to date that included patients with EGC who met the expanded indication criteria, with long-term follow-up. Third, the number of patients in the criterion II group was small. Kaplan-Meier analysis demonstrated no significant differences in OS and DSS between the two groups. However, because no mortality was seen in either group after matching, the Cox proportional hazard regression model could not be used to analyze OS and DSS. Fourth, this study did not assess quality of life after ESD and surgery.

In conclusion, to the best of our knowledge, this is the first study to compare long-term outcomes of ESD and surgery in EGCs meeting each criterion of the expanded indication. Considering comparable OS and DSS and the tolerable intensity of complications, ESD may be a reasonable alternative to surgery for EGCs that meet the expanded indication criteria.

\section{Compliance with ethical standards}

Conflict of interest The authors declare that they have no conflict of interest.

Funding This work was not supported by any direct or indirect funding. 
Human rights statement and informed consent All procedures followed were in accordance with the ethical standards of the Institutional Review Board of Asan Medical Center and with the Helsinki Declaration of 1964 and later versions. Informed consent or a substitute for it was obtained from all patients for being included in the study.

\section{References}

1. Ahn JY, Jung HY, Choi KD, Choi JY, Kim MY, Lee JH, et al. Endoscopic and oncologic outcomes after endoscopic resection for early gastric cancer: 1370 cases of absolute and extended indications. Gastrointest Endosc. 2011;74:485-93.

2. Choi KS, Jung HY, Choi KD, Lee GH, Song HJ, Kim DH, et al. EMR versus gastrectomy for intramucosal gastric cancer: comparison of long-term outcomes. Gastrointest Endosc. 2011;73:942-8.

3. Japanese Gastric Cancer Association. Japanese gastric cancer treatment guidelines 2014 (ver. 4). Gastric Cancer. 2017;20:1-19.

4. Choi IJ, Lee JH, Kim YI, Kim CG, Cho SJ, Lee JY, et al. Longterm outcome comparison of endoscopic resection and surgery in early gastric cancer meeting the absolute indication for endoscopic resection. Gastrointest Endosc. 2015;81(333-41):e1.

5. Fukunaga S, Nagami Y, Shiba M, Ominami M, Tanigawa T, Yamagami H, et al. Long-term prognosis of expanded-indication differentiated-type early gastric cancer treated with endoscopic submucosal dissection or surgery using propensity score analysis. Gastrointest Endosc. 2017;85:143-52.

6. Pyo JH, Lee H, Min BH, Lee JH, Choi MG, Lee JH, et al. Longterm outcome of endoscopic resection vs. surgery for early gastric cancer: a non-inferiority-matched cohort study. Am J Gastroenterol. 2016;111:240-9.

7. Abe S, Oda I, Nakajima T, Suzuki H, Nonaka S, Yoshinaga S, et al. A case of local recurrence and distant metastasis following curative endoscopic submucosal dissection of early gastric cancer. Gastric Cancer. 2015;18:188-92.

8. Fujii H, Ishii E, Tochitani S, Nakaji S, Hirata N, Kusanagi H, et al. Lymph node metastasis after endoscopic submucosal dissection of a differentiated gastric cancer confined to the mucosa with an ulcer smaller than $30 \mathrm{~mm}$. Dig Endosc. 2015;27:159-61.

9. Lee SH, Jee SR, Kim JH, Seol SY. Intramucosal gastric cancer: the rate of lymph node metastasis in signet ring cell carcinoma is as low as that in well-differentiated adenocarcinoma. Eur J Gastroenterol Hepatol. 2015;27:170-4.

10. Min BH, Kim ER, Kim KM, Park CK, Lee JH, Rhee PL, et al. Surveillance strategy based on the incidence and patterns of recurrence after curative endoscopic submucosal dissection for early gastric cancer. Endoscopy. 2015;47:784-93.

11. Charlson ME, Pompei P, Ales KL, MacKenzie CR. A new method of classifying prognostic comorbidity in longitudinal studies: development and validation. J Chronic Dis. 1987;40:373-83.

12. Dindo D, Demartines N, Clavien PA. Classification of surgical complications: a new proposal with evaluation in a cohort of 6336 patients and results of a survey. Ann Surg. 2004;240:205-13.

13. Hamilton SR, Aaltonen LA ; (2000) Pathology and genetics of tumours of the digestive system. IARC Press, Lyon

14. Park CH, Shin S, Park JC, Shin SK, Lee SK, Lee YC, et al. Longterm outcome of early gastric cancer after endoscopic submucosal dissection: expanded indication is comparable to absolute indication. Dig Liver Dis. 2013;45:651-6.

15. Hasuike N, Ono H, Boku N, Mizusawa J, Takizawa K, Fukuda $\mathrm{H}$, et al. A non-randomized confirmatory trial of an expanded indication for endoscopic submucosal dissection for intestinal-type gastric cancer (cT1a): the Japan Clinical Oncology Group study (JCOG0607). Gastric Cancer. 2017;. doi:10.1007/ s10120-017-0704-y.

16. Kim YI, Kim YW, Choi IJ, Kim CG, Lee JY, Cho SJ, et al. Longterm survival after endoscopic resection versus surgery in early gastric cancers. Endoscopy. 2015;47:293-301.

17. Ahn JY, Park HJ, Park YS, Lee JH, Choi KS, Jeong KW, et al. Endoscopic resection for undifferentiated-type early gastric cancer: immediate endoscopic outcomes and long-term survivals. Dig Dis Sci. 2016;61:1158-64.

18. Hahn KY, Park JC, Kim EH, Shin S, Park CH, Chung H, et al. Incidence and impact of scheduled endoscopic surveillance on recurrence after curative endoscopic resection for early gastric cancer. Gastrointest Endosc. 2016;84(628-38):e1.

19. Kato M, Nishida T, Yamamoto K, Hayashi S, Kitamura S, Yabuta $\mathrm{T}$, et al. Scheduled endoscopic surveillance controls secondary cancer after curative endoscopic resection for early gastric cancer: a multicentre retrospective cohort study by Osaka University ESD study group. Gut. 2013;62:1425-32. 